\title{
Enquête
}

Archives de la revue Enquête

3 | 1996

Interpréter, Surinterpréter

\section{Métaphores conventionnelles et métaphysiques anthropologiques}

La problématique de la traduction culturelle

Conventional metaphors and anthropological metaphysics. The problematic of

cultural translation

\section{Roger M. Keesing}

Traducteur : André Mary

\section{(2) OpenEdition}

\section{Journals}

Édition électronique

URL : http://journals.openedition.org/enquete/583

DOI : 10.4000/enquete.583

ISSN : 1953-809X

Éditeur :

Cercom, Éditions Parenthèses

Édition imprimée

Date de publication : 1 novembre 1996

Pagination : 211-238

\section{Référence électronique}

Roger M. Keesing, " Métaphores conventionnelles et métaphysiques anthropologiques », Enquête [En ligne], 3 | 1996, mis en ligne le 11 juillet 2013, consulté le 02 mai 2019. URL : http:// journals.openedition.org/enquete/583 ; DOI : 10.4000/enquete.583 


\title{
Métaphores conventionnelles et métaphysiques anthropologiques
}

\author{
La problématique de la traduction culturelle \\ Conventional metaphors and anthropological metaphysics. The problematic of \\ cultural translation
}

Roger M. Keesing

Traduction : André Mary

1 Roger M. Keesing est né en 1935. Il a suivi ses études aux universités de Stanford puis de Harvard, ce qui en fait un anthropologue de formation et de culture américaine. Après neuf ans d'enseignement à l'université de Californie (Santa-Cruz), de 1965 à 1974, il est nommé professeur d'anthropologie à l'Institut des Hautes Études de l'université nationale d'Australie où il exercera son activité d'enseignement et de recherche jusqu'à sa mort. Son ceuvre associe de façon exemplaire un engagement continu dans l'enquête de terrain, dont témoigne l'étude monographique qu'il a consacrée aux Kwaio de Malaita dans les îles Salomon et les nombreuses missions effectuées dans cette région à partir de 1962, et un souci de réflexion épistémologique sur les données accumulées, nourri de la connaissance des recherches les plus récentes dans le domaine de la linguistique, de la pragmatique et de l'anthropologie cognitive. Après sa thèse sur le mariage et la parenté dans la société Kwaio, il publie plusieurs articles sur la chefferie traditionnelle et le devenir du big man dans l'espace politique moderne, notamment à partir de l'histoire de vie d'un leader politique local'. Ses travaux sur la langue et la grammaire sont au centre de ses préoccupations, et son ouvrage majeur sur le système religieux des Kwaio est en fait l'étude cognitive des assomptions sur le monde et des prémisses culturelles qui sous-tendent les manières de parler indigènes ${ }^{2}$. Combinant une anthropologie culturelle critique et une analyse micropolitique des conflits locaux, R. M. Keesing s'intéresse aussi bien aux regards croisés des Britanniques et des indigènes sur les conflits de l'époque coloniale ${ }^{3}$ qu'au point de vue des femmes de ces sociétés, à la manière dont leurs récits autobiographiques éclairent leur rapport au pouvoir et à la parole ${ }^{4}$. Signalons enfin ses réflexions novatrices sur le statut de la "coutume» au sein même du mouvement de réinvention de la tradition et des luttes pour la revendication de l'autonomie culturelle. Impossible selon lui d'étudier la culture comme stock de connaissances et grammaire de règles sans s'interroger sur sa 
distribution inégale auprès de ceux qui en vivent et contribuent à la définir autant qu'à la contrôler.

2 La dimension polémique du débat sur la surinterprétation (ou la rnésinterprétation) du mana et de la stigmatisation de la théologie culturelle des anthropologues du symbolique, qui a contribué à faire connaitre les écrits de Keesing dans la communauté scientifique, ne doit pas faire oublier le socle des préoccupations cognitives et sociolinguistiques de l'auteur ${ }^{6}$ : quel rapport y a-t-il entre les manières de parler des gens, leurs présupposés sur le monde et leur expérience subjective? C'est par le détour de recherches savantes sur le rôle des schèmes métaphoriques dans la mise en forme de l'expérience que se trouve ici posé le problème des dérives interprétatives de l'activité ordinaire de traduction culturelle qui est au cœur du métier d'ethnographe. La quête du sens que poursuit une anthropologie dite "symboliste" comporte sans doute plus d'effet surinterprétatif que la question de savoir qui crée et manipule les significations culturelles (encore que...), mais il va de soi, aux yeux de Keesing, que la question du statut ambigu des formes symboliques, et, notamment, de l'usage différencié des schèmes métaphoriques qui informent la vision du monde et l'expérience des sujets d'une culture donnée, ne peut rester pour l'anthropologue un point aveugle. L'autoréflexion (renforcée par le "nous, les anthropologues ») et l'appel à l'ouverture d'un véritable champ de recherche sont aussi importants ici que la stigmatisation de l'autre. La mise en évidence de la surinterprétation passe de toute façon par un travail de réinterprétation et par une sorte d'anthropologie symbolique élargie et renouvelée.

3 Le cheminement du présent article est très significatif des difficultés de la posture adoptée. On commence par marquer l'écart entre l'évidence la plus apparente (en l'occurrence l'évidence linguistique) et les fictions métaphysiques de la religion des anthropologues, ou plus subtilement entre les présupposés " métaphysiques » du parler ordinaire et les élaborations " chargées de sens » des métaphysiques savantes, et, chemin faisant, la frontière se trouble: on découvre que la parole indigène n'exclut pas la possibilité de l'exégèse, qu'il arrive que les conventions métaphoriques engendrent des croyances "réelles" ou même que le mana soit "véritablement" réifié et traité comme une substance. En un mot, les autres aussi surinterprètent, ou plus exactement certaines sociétés, certaines langues, certains groupes ou agents, dans un certain contexte, et cela aussi fait partie de l'évidence ethnographique. La conséquence méthodologique est importante puisqu'il s'agit moins d'appeler à faire table rase de données ethnographiques imaginaires, ou à s'y complaire, qu'à mieux les situer et les contextualiser en s'interrogeant notamment sur les conditions qui conduisent certaines sociétés ou certains agents à investir l'activité interprétative comme enjeu de pouvoir.

4 Le problème anthropologique majeur sur lequel débouche le questionnement ethnographique de Keesing est redoutable: dans quelle mesure les schèmes métaphoriques et les expressions linguistiques qui informent notre expérience cognitive et émotionnelle du monde (et celle des autres) peuvent-ils être considérés comme une description adéquate de ce que nous (et les autres) pensons et ressentons "réellement"? La leçon de l'auteur est celle de la prudence et même du scepticisme méthodique, une attitude paradoxalement encouragée par la multiplication des années de terrain'. Mais nul doute en revanche qu'il existe pour lui, en contrepoint de nos fictions langagières, une "réalité» de l'expérience, et même, au-delà d'une certaine variabilité des formes culturelles - largement exagérée à son avis par le paradigme culturaliste -, une étonnante invariance des schèmes qui président à la symbolisation de l'expérience humaine. L'anthropologue n'a pas à renoncer à l'ambition de savoir ce que les hommes pensent "réellement » et ressentent " profondément ", à la seule condition de ne pas prêter à leurs manières de parler plus de réalité et de profondeur qu'elles n'en ont. Le pari est difficile mais il faut bien qu'il y ait un "fond de réalité" quelque part qui remonte à la surface des choses sons la forme d'une "évidence flagrante» si l'on 
veut échapper à la fois aux excès de sens et au solipsisme relativiste.

ANDRÉ MARY

Si un ethnographe rencontre des gens qui parlent comme si l'univers était dominé par une énergie dynamique, une sorte de fluide, ou comme si le ventre était le centre des émotions, ou encore comme si les arbres pouvaient exprimer des idées, comment faut-il interpréter de telles manières de parler? Doivent-elles être comprises comme des expressions d'idées métaphysiques ou tout simplement comme des métaphores conventionnelles, des figures du discours, des tropes? Mon argument sera que nous autres, les anthropologues, sommes des professionnels du marché de l'exotisme. Si les significations sont ambiguës, nous avons tendance à choisir des lectures qui présentent les populations que nous étudions comme ayant une culture, des croyances, des symboles étonnamment différents des nôtres. Lorsque notre évidence se nourrit de la substance du langage (et lorsque notre connaissance des langues du terrain n'est pas à la hauteur de la compétence indigène), nous pouvons projeter dans les paroles des autres des pertinences métaphysiques qui n'y sont pas du tout.

6 Je me propose d'explorer les implications épistémologiques des recherches récentes sur la métaphore conventionnelle pour l'entreprise anthropologique. $\mathrm{Si}$, comme le soutiennent un certain nombre d'écrits récents sur les métaphores, la plupart d'entre elles ne sont ni "vivantes » ni "mortes ", mais tout simplement conventionnelles ${ }^{9}$, basées sur des «cadres » culturellement établis ${ }^{10}$ ou sur des «modèles culturels ${ }^{11}$ » - alors il en est de même du parler ordinaire dans la jungle, le désert, ou dans les villages du monde paysan où nous faisons notre travail de terrain.

7 En mettant en évidence la cohérence des paradigmes de la métaphore conventionnelle, la manière systématique dont ils suggèrent des relations entre les domaines et la façon pénétrante dont ils structurent notre langage et peuvent canaliser et informer notre expérience, la recherche récente lance un défi aux anthropologues invités à dresser la carte des schèmes métaphoriques des populations non occidentales. Notre interprétation et nos analyses de la métaphore ont été, mises à part quelques notables exceptions, fragmentaires et partielles; comme le laisse entendre Salmond ${ }^{12}$, une exploration beaucoup plus systématique s'impose.

L'essentiel de mes préoccupations s'inscrit dans une autre orientation. Les ethnographes peuvent souvent avoir projeté sur les manières de parler des autres des croyances métaphysiques et des cosmologies que celles-ci semblaient, à tort, impliquer. Avons-nous inventé des cosmologies, des théologies, des croyances, construites à partir des métaphores des autres? Avons-nous, ethnographes, agi comme des théologiens inventant des théologies fictives?

\section{L'anthropologue théologien}

Les ethnographes qui travaillent dans les sociétés tribales rencontrent de façon caractéristique des gens dont la cosmologie est en grande partie présente à l'état implicite dans leur pratique rituelle et leur conduite au quotidien. Habituellement peu d'informateurs, s'il en est, articulent à leur intention une explication globale et cohérente 
de leurs systèmes de croyances et de leurs significations rituelles. Il existe de notables exceptions parmi les peuples tribaux, telle que les Iatmul de Nouvelle-Guinée, où l'érudition théologique est une source de pouvoir mondain ${ }^{13}$. Et l'ethnographe qui a de la chance peut rencontrer un philosophe populaire, comme Ogotemmeli ou Muchona le Frelon, qui étale toute une vision globale, riche et cohérente, de « son » monde ${ }^{14}$. Une part du danger que représente la théologie culturelle vient précisément de notre propension à rechercher le philosophe populaire le plus doué et le plus savant, et à élever ce qui peut être une synthèse ou une extrapolation personnelle jusqu'au royaume des «symboles culturels ».

10 Je me sens encore plus concerné par la tentation de l'anthropologue de prendre les pièces et morceaux présents dans la pratique rituelle et dans ce que les acteurs indigènes font et disent, et de construire à partir de ces éléments une philosophie cohérente qu'aucun informateur n'a lui-même conçue. L'ethnographe joue le rôle de théologien culturel, suppléant aux éléments qui manquent, assemblant les fragments épars, transformant en apparence l'implicite en explicite.

11 Il existe deux modes de distorsion virtuelle en la matière. En premier lieu, par le fait de rendre cohérent ce qui est fragmentaire, l'ethnographe peut gommer des contrastes significatifs entre, d'un côté, les visions du monde pragmatiques, partielles et sociocentriques, caractéristiques des populations tribales (plus concernées par l'agir dans le monde que par la tendance à l'expliquer systématiquement) et, de l'autre, les théologies culturelles cohérentes qui sont le propre des sociétés stratifiées en classes comportant des théologiens professionnels. Une cosmologie cohérente fallacieuse, inventée par l'ethnographe à partir d'une vision du monde partielle et pragmatique, peut, comme Brunton ${ }^{15}$ l'a récemment suggéré dans le cadre de la Mélanésie, dissimuler des écarts qui appellent une explication théorique.

Le second problème est plus sérieux. L'ordre manquant complété par l'analyste peut être un faux. Ce qui semble impliqué par une évidence de surface peut être une inférence fallacieuse de l'ethnographe. Un exemple frappant nous est fourni par le réexamen récent auquel se livre Needham ${ }^{16}$ de la question de la chasse aux têtes dans certaines régions de l'Asie du Sud-Est. Les premiers ethnographes, principalement allemands, ont relevé le lien entre la prise de têtes et les profits qui étaient censés revenir aux chasseurs de têtes, particulièrement pour la fertilité des récoltes. Les Européens en ont déduit que le lien entre la prise de tête et les bénéfices mondains est un médium invisible, une "âmesubstance ", une force vitale de la personne, à la fois sociale et individuelle, située dans la tête et qui peut être acquise par une personne qui prend cette tête. La chasse aux têtes est ainsi motivée par la poursuite de cette "âme substance " qu'il s'agit de s'approprier. Needham accumule les évidences qui montrent que la plupart de ces chasseurs de têtes d'Asie du Sud-Est n'ont en fait aucune idée de l'«âme-substance »: cet hypothétique médium spirituel est un faux ingrédient créé par les ethnographes pour alimenter un lien causal entre la prise de tête et les bénéfices qui s'en suivent. Les réflexions de Needham ${ }^{17}$ méritent d'être considérées :

«La fabrication de l'idée d'âme-substance n'est pas suffisamment expliquée par le simple fait qu'elle se révèle utile pour l'analyse. [...] Nous avons besoin de comprendre [...] pourquoi précisément cette idée particulière en est venue à être formulée dans ce but. La réponse peut être décelée, je pense, dans les images descriptives auxquelles les ethnographes ont typiquement recours dans leurs tentatives pour traduire ce qu'ils conçoivent comme l'œuvre d'un agent causal. Kruyt introduit l'idée d'une "force de vie", d'abord pensée comme un "fluide" puis 
comme une "substance"; Elshout fait référence à une "énergie" magique [...] et Izikowitz, parlant du klpu chez les Lamet, évoque "une sorte de fluide, comme l'électricité". [...] Mais tous ces termes appartiennent en fait à un idiome scientifique dérivé de la physique : électromagnétisme, hydraulique, mécanique. Ils ont été cités par les ethnographes parce qu'il fallait rendre compte en termes de causalité de certains effets. »

13 Needham pointe une influence similaire des images dérivées de la physique classique dans les interprétations anthropologiques des grands sociologues français (Durkheim, Mauss, et les autres). Mauss, par exemple, s'appesantit sur l'« esprit du don »: "Quelle force y a-t-il dans la chose donnée, demande-t-il, qui fait que celui qui la reçoit la rend? ». De telles réifications et métaphysiques fallacieuses ont la vie dure. Je pense que nous, les ethnographes, perpétuons ce genre de choses et continuons dans le même temps à les inventer.

Un exemple tout aussi frappant que j'ai eu l'occasion d'explorer moi-même nous ramène au fameux concept océaniste de mana. Un grand nombre de faits linguistiques et ethnographiques ${ }^{18}$ indiquent que dans les premières langues de l'Océanie, dont sont issues les langues de la Mélanésie orientale, des Fidji, de la Micronésie centrale et de la Polynésie, mana correspondait, selon l'usage autorisé, à un verbe d'état dont les significations étaient " être efficace, être vrai, être accompli, être puissant ", avec l'idée qu'une telle efficacité et une telle puissance étaient le produit d'une bénédiction ou d'une protection, ou encore de la transmission d'une force par les ancêtres ou d'autres esprits. Le terme était aussi utilisé comme verbe transitif ( " mana-iser ma formule magique », ou «mana-iser mon jardin » ou "mana-iser ma pirogue de guerre ») et comme un verbe intransitif («mana pour moi », « mana, mana, mana! ») dans les prières où l'on s'adresse aux esprits. Finalement, mana était utilisé comme un nom morphologiquement abstrait et indistinct dérivé d'un verbe d'état. Dans certaines langues inscrites dans cette filiation, ce nom abstrait et dérivé est affecté d'un suffixe marquant son usage nominal. C'est ce cas de figure (présent dans la langue Kwaio et dans d'autres langues Malaita) - et la découverte rétrospective de mes propres erreurs de traduction - qui m'a conduit à mener une analyse comparative.

Le triple usage du terme mana en tant que verbe d'état, verbe d'action et nom abstrait est dominant dans la Mélanésie orientale, et répandu en Polynésie et en Micronésie. Ce qui est surprenant, lorsque l'on retourne aux textes, c'est la théologie fallacieuse qu'on a greffée sur le mana en tant que substance diffuse, médium invisible du pouvoir que les hommes recherchent auprès des fantômes, des esprits et des dieux. Une telle métaphysique semble, comme l'«âme-substance » des chasseurs de têtes, être pour l'essentiel une création de théologiens européens et non d'autochtones. Les Mélanésiens s'adressant aux esprits pour l'efficacité (et l'attribution de puissances aux uns et aux autres) sont conçus comme étant en situation de négociation ou de possession d'un médium invisible de pouvoir spirituel. La plupart du temps, c'est un pur contresens qui a conduit à la faute. Dès l'époque de Codrington, lorsque les anthropologues ont découvert que mana était un nom, nous avons en fait enregistré le terme utilisé pour qualifier un état ou comme verbe, et nous l'avons traduit comme un nom. "Mana pour moi », dit un Mélanésien à son ancêtre en prière. « Donne moi le mana », traduit l'anthropologue.

16 Mais dans certaines parties de la Mélanésie, et dans beaucoup de régions de la Polynésie, mana en tant que nom abstrait dérivé («efficacité, puissance ») était au moins partiellement substantivé ou concrétisé. Mana devient, au moins métaphoriquement, quelque chose dont les individus disposent plus ou moins. J'ai émis l'hypothèse que cette 
élaboration théologique était un produit d'un certain type de hiérarchie politicoreligieuse de l'Océanie ${ }^{19}$. Il est difficile pourtant, avec seulement quelques textes originaux crédibles, de faire la part entre théologie indigène et mésinterprétation européenne. Douglas Oliver ${ }^{20}$, à qui je dois une part de mon scepticisme ethnographique, a noté, en référence à Tahiti, le problème de la surinterprétation et de la création d'une métaphysique fictive :

\begin{abstract}
«À mon avis, les données concernant les îles de la Société ne permettent pas de soutenir la thèse de E.S. C. Handy ${ }^{21}$ selon laquelle ces Polynésiens conçoivent l'univers comme un dynamisme psychique qui se manifeste lui-même physiquement. [...] Aucun texte enregistré ou aucun commentaire sur les conduites qui me soit connu, ne fournit la moindre évidence que les habitants de ces îles cultivent une telle vision générale et englobante, énergique et animiste, de leur univers. [...] Je crois que le point de vue ci-dessus énoncé, d'une compréhension si hautement raffinée, est le produit de la pensée philosophique de l'ethnologue et non, même en termes implicites, des insulaires eux-mêmes. »
\end{abstract}

Nous retrouvons, comme Needham le notait pour l'«âme-substance », la tendance à imputer aux populations non occidentales des cosmologies totalisantes et universalistes, caractérisées par la terminologie de l'électromagnétisme, de la mécanique et de l'hydraulique, héritée de la physique classique.

Comment pouvons-nous éviter la mésinterprétation ou la surinterprétation de ces systèmes conceptuels populaires? Nous avons besoin avant tout de nous attaquer à notre tâche d'interprétation en la traitant carrément comme une tâche de traduction culturelle, une tâche pleine de difficulté et de danger.

\title{
Réification et distorsion dans la traduction ethnographique
}

Une des erreurs les plus répandues que j'ai rencontrée dans les interprétations anthropologiques du mana réside dans le fait de traduire un état ou un verbe comme s'il s'agissait d'un nom. De telles erreurs de la part de spécialistes travaillant dans l'ombre de Codrington $^{22}$ sont peut-être compréhensibles, mais une telle mésinterprétation de termes décrivant des processus ou des états par le recours à des substantifs de la langue anglaise semble être un problème plus général. Un premier pas vers une traduction culturelle moins soumise à distorsion serait d'être fidèle à l'évidence linguistique à portée de main et d'être constamment attentif aux dangers (auxquels la langue anglaise, semble-t-il, nous prédispose spécialement) de la réification et de la fausse substantialisation, ou encore d'attribuer à un monde de relations et de processus ce que le philosophe des sciences Campbell appelle l'« entitivitée ${ }^{3} »$.

Une traduction erronée peut engendrer non seulement des entités fictives mais également de faux problèmes d'analyse. Le terme océaniste d'origine austronésienne tapu / tabu, qui, comme le mana, a occupé une place dominante dans l'anthropologie du Pacifique et dans le métalangage de l'analyse comparative, est un cas intéressant. Tapu et ses formes apparentées sont, selon les canons de la langue, des verbes d'état. Qu'il s'agisse des commentaires sur les coutumes polynésiennes ou mélanésiennes ou, plus généralement, du discours anthropologique («le tabou de l'inceste »), ce mot qui désigne un état a été traduit comme s'il était question d'un nom, dans la continuité d'un procès de réification et de distorsion qui commence ironiquement avec les voyages de $\operatorname{Cook}^{24}$. Les 
anthropologues (qui ont au moins la plupart du temps correctement noté la forme d'état comme étant première) ont souvent créé, par une traduction trompeuse, de pseudoproblèmes. La signification de base de tapu dans les deux langues mélanésiennes et polynésiennes semble être rendue au mieux par "off-limits », " défense d'entrer », tout simplement ${ }^{25}$. Cela permet de saisir la dimension relationnelle de tapu, sur un mode que « sacré » ou « interdit » exclut; et cela nous libère de l'apparente contradiction entre la valeur positive de la sacralité et la valeur négative de la « pollution » et de l'interdiction. Quelque chose qui est tapu est ainsi «off-limits ", et cela implique inévitablement : 1) un agent ;2) une perspective ; 3) un contexte.

Quelque chose est " off-limits", seulement si quelque agent (humain ou non humain) le définit en tant que tel. Cet agent peut être un dieu, les ancêtres, ou un chef (quasi-dieu). Dans beaucoup de langues d'Océanie, il se peut aussi que ce soit un parent disant à un enfant de ne pas faire quelque chose ${ }^{26}$. Quelque chose est « off-limits ", tapu, seulement à partir d'une perspective donnée. Ce qui est "off-limits» pour une personne ou une catégorie de personnes peut être "permis» (souvent rendu par noa ou des formes similaires) ou même prescrit pour une autre personne ou catégorie de personnes. Une case menstruelle peut être tabu du point de vue privilégié des hommes; au regard de l'avantage d'une femme qui a ses règles, il serait tabu de se trouver partout ailleurs que dans ce lieu. De même, une maison des hommes peut être tapu pour les femmes, noa pour les hommes ; une perspective est toujours en cause. Quelque chose qui est "off-limits", tapu, l'est toujours pour quelqu'un, et non en soi ou par soi. En définitive, être tapu, offlimits, suppose un contexte. Une place, un acte ou une chose, qui est tapu cet après-midi, du point de vue de certaines gens et dans le contexte d'une circonstance ou d'un rituel particulier, peut être noa (ou tapu) pour une population différente demain. Se trouve ainsi souligné ce pourquoi les traductions de tapu par «sacré" ou par «interdit» sont trompeuses.

La sainteté est une qualité que nous imaginons inhérente aux gens, aux lieux ou aux choses de manière absolue et non contextuelle. Or même la qualité tapu d'un prêtre ou d'un chef dans la Polynésie orientale était contextuelle et relative, bien que la fusion du pouvoir politique et de l'intercession sacerdotale auprès des dieux semble avoir conduit à des élaborations théologiques des notions de tapu aussi bien que de mana. Sahlins ${ }^{27}$, dans son livre récent sur Hawaï, note les élaborations de la « tapuité » qui émane des dieux et qui entoure les chefs, et il y va de commentaires sardoniques sur la manière dont les Hawaïens modernes écrivent "kapu» sur les clôtures, comme si le mot signifiait simplement "entrée interdite, propriété privée ». Sans doute Sahlins a-t-il raison de souligner qu'à Hawaï un terme au départ imprégné de significations religieuses a fini par se séculariser. Cependant et non sans ironie, « off-limits » a été la racine sémantique de tapu pendant au moins quatre cents ans, et cela dans la transparence pour les locuteurs de l'Océanie tout au long de cette période et sur une vaste aire géographique. Nous ne pouvons utiliser de meilleure métaphore pour traduire le sens de tapu que celle d'une clôture interdisant l'accès, que cette clôture ait été érigée par des dieux, des chefs ou de simples mortels. Si nous interprétons tapu comme "off-limits" (sans aucune autre préconception, que celle-ci concerne le fait que la « clôture » ait été érigée par des dieux ou des ancêtres ou qu'elle ait à voir avec les qualités inhérentes à l'acte, l'objet ou la personne qui est mise à part), nous échappons au pseudo-problème sur lequel Steiner et bien d'autres se sont appesantis, à savoir pourquoi le terme se réfère à la fois à la sainteté 
et à ce qui est prohibé ou pollué. Il fait bien référence aux deux valeurs et en même temps à aucune.

Une première étape urgente vise donc les traductions soignées, attentives à ne pas concrétiser faussement, à ne pas créer des substantifs inexistants à partir de relations, d'états ou de processus. Cela ne veut pas dire que nous, les analystes, ne pouvons pas ou ne devons pas aller au-delà des catégories indigènes, mais une première tâche est incontestablement d'en rendre compte aussi fidèlement que nous le pouvons.

Les peuples de l'Océanie (certains d'entre eux, au moins) parlent de fait comme si les individus qui ont toujours $\mathrm{du}$ succès, qui sont manifestement puissants ou héréditairement sacrés, " avaient du mana ». Au sens littéral, ils semblent vouloir dire que le mana, dont les gens disposent plus ou moins, est un médium ou un instrument de pouvoir, de succès et de sacralité. Et cela m'amène à la question de la métaphore conventionnelle.

\section{De la métaphore conventionnelle}

Le rôle influent de la métaphore conventionnelle dans le langage ordinaire a été très fortement démontré par Lakoff et Johnson. Ils mettent en avant ce qu'ils appellent une théorie "expérientielle» de la métaphore. Les métaphores conventionnelles ont un caractère systématique (nous pourrions dire paradigmatique) en ce qu'elles suggèrent un univers dans lequel on parle d'un domaine de réalité dans les termes d'un autre. Ainsi les métaphores de la spatialisation décrivent les réalités temporelles, émotionnelles, ou expérientielles en termes d'espace. Dans les métaphores ontologiques, les forces ou entités naturelles sont personnifiées ou les abstractions sont concrétisées, comme dans les métaphores qui assimilent les théories à des édifices construits sur des fondations. Les "métaphores structurales" proposent une homologie de structure entre une source de référence et une réalité visée, comme dans le cas de l'équivalence que nous établissons entre le débat rationnel et la guerre.

Lorsque l'on considère les schèmes métaphoriques qui traversent une série de langues, nous pouvons nous attendre à trouver à la fois une diversité culturelle et peut-être une étonnante régularité. Ainsi toutes les langues, au moins virtuellement, décrivent les relations dans le temps en termes de relations dans l'espace. Les émotions sont décrites en référence aux couleurs («bleu », « vert d'envie », « voir rouge »). D’autres métaphores spatiales très répandues, peut-être universelles, caractérisent le monde physique à partir des termes dans lesquels les hommes le découvrent au niveau de la perception, comme si la terre était plate, le soleil et la lune se déplaçaient alors que la terre restait au repos (le soleil se "lève » et se " couche »), même si cela n'est pas en accord avec les connaissances astronomiques modernes. Dans ces domaines, les schèmes métaphoriques sont beaucoup plus semblables d'une langue à l'autre que nous pourrions le penser. La vie est considérée comme une marche en ligne droite, un chemin sur lequel le futur se situe vers l'avant et le passé en arrière ${ }^{28}$. Les métaphores reposant sur les parties du corps diffèrent beaucoup moins que nous pourrions le croire d'une langue à l'autre ${ }^{29}$. Les langues contiennent cependant de fait des systèmes de métaphores qui sont spécifiques à chaque culture (sans être nécessairement uniques). Lakoff et Johnson illustrent ce point, dans le cas de l'anglais, par le système de métaphores qui assimile le temps à la monnaie : nous gagnons du temps, nous le gaspillons, nous le budgétisons, et ainsi de suite. 
27 Les langues non occidentales peuvent user de schèmes métaphoriques différents des nôtres pour caractériser les émotions et les processus cognitifs. Lakoff et Kovecses ${ }^{30}$ analysent le modèle systématique par lequel, en anglais, la colère est assimilée à un liquide chaud contenu dans un récipient. Rosaldo ${ }^{31}$ explicite les usages de liget chez les Ilongot, un terme qui exprime également, bien que différemment, un système de schèmes métaphoriques des états émotionnels de la « colère ».

28 Ce qui est le plus intéressant sur le plan anthropologique à propos de tels modèles de métaphore c'est qu'il suggère un univers de discours métaphorique, au sein duquel on parle d'un domaine d'expérience dans les termes d'un autre. C'est cette suggestion d'un univers, formé par les métaphores d'une culture étrangère, qui pose un problème épistémologique particulier auquel nous n'avons pas accordé suffisamment d'attention. Considérons le cas de mana. Lorsqu'un Polynésien dit que les chefs ont du mana, et que c'est pour cela qu'ils sont tapu, faut-il pour autant imaginer que mana est une substance spirituelle influente qui se répand comme l'électricité, une substance dont les gens disposent plus ou moins, grâce aux dieux?

\section{La métaphore conventionnelle : quelle profondeur ? quelle pertinence?}

Avant que nous puissions poser le problème de la juste interprétation des modèles de métaphore conventionnelle des autres peuples pour les anthropologues, il est utile de considérer de plus près l'argumentaire de Lakoff et Johnson, en tant qu'il se réfère notamment à une ethnographie de la vision du monde codifiée dans la langue anglaise. Lakoff et Johnson s'appliquent à montrer que les métaphores vivent dans un autre monde, d'une autre manière, que celui ou celle que nous imaginons habituellement. Les métaphores conventionnelles dominent notre langage ordinaire; nous ne pouvons parler, nous ne pouvons penser qu'en elles et par elles ${ }^{32}$. Elles sont construites à partir de nos expériences les plus fondamentales, issues de corps et de cerveaux humains, d'un appareil sensible, situés dans un monde semblable aux nôtres; et dans le même temps, selon l'argumentaire de Lakoff et Johnson, nos manières conventionnelles de caractériser l'expérience subjective sont constitutives de l'expérience elle-même. Nous nous percevons comme des agents-dans-le-monde, en partie dans les termes métaphoriques conventionnels de l'agencéité et de la cause (et, comme l'a noté Whorf, sans doute également dans les termes de moules grammaticaux issus du langage). Nous sommes inextricablement situés dans un monde que le langage crée pour nous.

L'argument lancé dans Metaphors We Live By est encore à développer et à raffiner, ses implications étant toujours à explorer. Dans leur analyse de la conception anglaise implicite de la colère, Lakoff et Kovecses montrent qu'un schème métaphorique central qui assimile celle-ci à un fluide retenu dans un contenant, en liaison avec une série de métaphores associées, oriente ou dessine un scénario prototypique de la colère, de ses raisons et de son déroulement: "Ceci nous permet de montrer de manière précise comment les différentes métaphores sont reliées les unes aux autres, et comment elles fonctionnent ensemble pour aider à caractériser un concept particulier. C'est une chose que Lakoff et Johnson étaient incapables de faire. »

31 Lakoff et Kovecses montrent que l'ontologie de la colère mise en scène dans ce scénario prototypique est «en général constituée par métaphore"; mais ces métaphores constitutives, caractérisant le déroulement de la colère en termes de limite, de force, de 
contrôle, etc., sont extraites de domaines relativement abstraits, de source " supérieure ». Par contraste :

«Les métaphores principales qui dessinent l'ontologie de la colère - LIQUIDE CHAUD, FOLIE, FEU, FARDEAU, COMBAT - apparaissent comme étant des concepts de base, c'est-à-dire des concepts qui sont liés plus directement à l'expérience, des concepts qui sont riches en information et en images mentales conventionnelles. [...] Une grande partie de notre compréhension de la colère passe par ces métaphores de base. [...] Sans elle notre compréhension de la colère serait extrêmement appauvrie."

Bien qu'ils prolongent le schéma conceptuel proposé par Lakoff et Johnson bien au-delà de ce qu'il était, ces auteurs s'accordent à reconnaître que des questions cruciales subsistent. Étant donné que l'usage linguistique est essentiellement constitué par des schèmes métaphoriques sous-jacents dont il est également l'expression systématique, Lakoff et Kovecses s'interrogent sur ce que sont les ressorts psychologiques et expérientiels de tels schèmes métaphoriques.

« Notre méthodologie ne nous permet pas d'en dire plus sur le statut psychologique $\mathrm{du}$ modèle [de la colère] que nous avons découvert. Quelle est la part de ce modèle dans la manière dont les gens comprennent de fait la colère ? Est-ce que les gens se basent réellement dans leurs actions sur ce modèle ? Quelle importance a-t-il, s'il en a une, dans ce que les gens croient consciemment? Et plus curieusement, le modèle a-t-il un quelconque effet sur ce que les gens ressentent?»

Ils observent qu'il n'est nullement certain que le modèle de la colère qu'ils explicitent soit également pertinent pour tous les locuteurs de langue anglaise, qu'il ait les mêmes significations pour eux.

Lakoff et Kovecses, en prolongeant l'analyse de Lakoff et Johnson, éclairent la mise en forme culturelle et la nature systématique d'un modèle de la colère construit en anglais, un modèle par conséquent que nous, les anthropologues, pourrions nous attendre à trouver différent des modèles de la " colère » et des émotions associées qui sont codifiés dans les autres langues et cultures. Les analyses de Rosaldo ${ }^{33}$ consacrées au terme liget chez les Ilongot des Philippines et les analyses de Lutz ${ }^{34}$ portant sur les conceptualisations culturelles des émotions chez les Ifaluk confirment amplement ces hypothèses.

Mais la question de savoir dans quelle mesure les métaphores conventionnelles sont constitutives de l'expérience reste incertaine. Il paraît vraisemblable que beaucoup d'entre elles le sont, et qu'elles informent et canalisent de manière pénétrante notre pensée. Mais beaucoup d'entre elles peuvent ne pas être aussi profondément pertinentes. On ne peut non plus admettre tranquillement, comme Lakoff et Kovecses nous en avertissent, que sous prétexte que tous les locuteurs indigènes utilisent les mêmes expressions linguistiques conventionnelles, celles-ci ont les mêmes significations pour chacun d'entre eux.

Considérons par exemple la manière dont les locuteurs anglais parlent du cœur dans leurs métaphores conventionnelles. Je n'ai aucun doute sur le fait que Lakoff et Kovecses puissent mettre en évidence, avec une habileté équivalente, de nombreuses structures faisant système dans notre façon de parler des cœurs. En tant que formule systématique permettant d'évoquer des états intérieurs ayant les propriétés d'une entité, la métaphore du cœur nous est très utile ; ses qualités sont décrites de manière significative en termes sensibles : léger ou lourd, le cœur se soulève ou se serre, il est pur, solide, ou brisé, dur ou tendre. Les processus et les expériences affectifs et cognitifs associés au cœur dans les métaphores conventionnelles sont en contraste avec l'aspect purement intellectuel de 
ceux qui se réfèrent aux choses de la «tête ». Mais le cœur représente parfois la volonté et la résolution, parfois les émotions (particulièrement l'amour, la compassion, la sentimentalité), parfois la profondeur de l'engagement, parfois le profond, le fondamental, et l'indicible.

La question de savoir à quel organe physique on fait appel comme instrument métaphorique pour caractériser les émotions, les pensées et les sentiments les plus intenses, et les aspects les plus profonds et les plus stables de la volonté et du caractère, ne relève pas de l'arbitraire : il faut à ce sujet un organe principal, viscéral, et non un organe périphérique (il doit être physiquement "profond" aussi bien que central); idéalement se trouve écarté (peut-être) tout organe que nous guidons directement sur le plan physiologique ou que nous contrôlons volontairement. En cas de stress émotionnel nous faisons l'expérience d'une palpitation accélérée. Ainsi le cœur est un domaine source adapté, dont le choix ne relève pas du hasard, pour nos manières de parler des émotions, de la volonté, du caractère, etc. Et en un certain sens, les oppositions signifiantes tirées du langage du cœur sont à la fois pertinentes sur le plan de l'expérience et également formatrices et constitutives des voies par lesquelles nous interprétons notre être et celui des autres.

Mais au-delà du langage du cœur, le recours à l'anatomie des viscères comme domaine source ne consiste pas à attribuer à ce dernier les processus décrits à partir de ses termes. Le cœur n'est pas, pour nous, le « siège des émotions ». Notre explication populaire à ce sujet (intégrée dans de nombreux et savants volumes) est qu'à une certaine époque de notre passé, lorsque nous étions moins raffinés, nous, occidentaux, avions pour usage de croire que le cœur physique était le "siège des émotions "; nos métaphores sont tout ce qui reste de ces anciennes croyances. Les observations de MacCulloch ${ }^{35}$ dans l'Encyclopeedia of Religion and Ethics donnent forme à cette théorie populaire :

«Une conception du cœur qui en fait le siège de l'expérience religieuse, de l'émotion, de l'illumination et de la présence divine, est commune à la plupart des grandes religions. [...] Bien sûr, ce qui est ici une conception métaphorique du mot repose en fait sur des vues antérieures concernant le cœur physique en tant que tel.»

Dans la pensée primitive, le foie était sans doute considéré comme un siège originel de la vie, mais en général le cœur, au fur et à mesure que ses fonctions physiologiques étaient mieux comprises, a fini par être considéré de cette façon.

Une telle conception de nos métaphores comme résidus d'anciennes croyances désormais gelées, me semble être fondamentalement contredite par le travail de Lakoff et Johnson. Si les métaphores dominent notre langage, informant notre expérience tout en la stimulant, suggérant des homologies pertinentes (mais non en termes métaphysiques) entre des domaines source et des domaines cible, nous devons nous attendre à ce que la caractérisation métaphorique du monde soit un processus à l'œuvre dans toutes les langues. Nous ne pouvons présupposer que les façons de parler des autres peuples à propos des cœurs et des foies, du lever et du coucher du soleil, de la réussite et de l'échec, ont pour eux-mêmes des profondeurs de sens qualitativement différentes de celles qui sont les nôtres.

41 Les mêmes problèmes et les mêmes questions surgissent à propos des manières de parler qui sont moins clairement métaphoriques mais tout aussi conventionnelles. Considérons par exemple les façons de parler dont usent les locuteurs anglais pour évoquer la « chance» (luck). Le prototype du scénario dont dérivent les significations centrales de la 
« chance » (et également de la bonne fortune) est (je pense) un jeu à risque qui apparaît fondé sur le hasard mais qui en fait a de «lourdes » conséquences pour les participants, conséquences qui sont invisibles mais qui transparaissent dans les gains et les pertes. Le schéma métaphorique qui dérive de ce scénario prototypique suppose, comme je le pense, que : 1) La chance est une substance invisible dont les «joueurs » disposent plus ou moins; ainsi "certaines gens ont toujours de la chance ", "ma chance s'est évanouie ", "pas de chance ». 2) La chance, en tant que substance, présente deux variétés, la bonne chance et la mauvaise chance; ainsi «j'ai eu de la malchance aujourd'hui, mais de la veine hier », etc. 3) La chance est une personne qui s'active à contrôler le jeu ; ainsi « Dame fortune ", « la chance est avec moi », « la chance est de mon côté », « la fortune me sourit », « le coup de pouce du hasard », etc.

Mais qu'en est-il de la pertinence, pour les locuteurs anglais, de telles conventions dans les manières de parler? Tout d'abord, je pense que nous pouvons sans prendre de risque en déduire que les significations de la «chance " pour des locuteurs anglais différents varient considérablement. Beaucoup d'entre nous admettent que les conséquences des événements que nous assimilons à un jeu de la chance relèvent en fait du hasard. Nous faisons usage d'expressions linguistiques conventionnelles de la chance pour caractériser des résultats particuliers dans des circonstances particulières, parlant comme si les « jeux » de la vie avaient des conséquences déterminées sans «croire " pour autant qu'il en est réellement ainsi. Mais beaucoup d'entre nous peuvent aussi «croire» que les résultats sont déterminés. Lorsqu'un locuteur anglais dit que "Certaines personnes ont toujours de la chance ", il ou elle peut très bien être en train de faire une remarque sur l'inégalité des chances des gens dans la vie, ou de faire un commentaire (sardonique ou moqueur) à propos d'une situation particulière.

43 Les métaphores conventionnelles de la chance en tant que substance invisible sont ritualisées pour beaucoup de locuteurs anglais par des "porte-bonheur", tels que les pieds de lapin. Mais ceux qui ont des amulettes qui portent chance, ou les athlètes qui ont des crosses de golf ou des bandeaux porte-chance, peuvent (je le pense) avoir des notions très diverses (s'ils en ont) sur la manière dont ces objets sont reliés à ou influent sur les conséquences des événements.

44 Notre langage de la "chance" illustre des manières conventionnelles de décrire l'expérience de la vie en termes de jeu échappant au hasard, des manières qui sont incorporées dans la substance même des habitudes du langage ordinaire. Notre manière de parler de la " chance » n'a aucune relation précise avec des croyances métaphysiques concernant les forces qui contrôlent l'univers, au même titre que notre langage du « cœur » n'implique aucune physiologie de l'esprit ou de l'émotion. La façon dont les gens qui parlent anglais se représentent effectivement le monde "à l'œuvre " n'est ni déterminée par ni déductible de tels modes conventionnels du langage. Dans les conventions métaphoriques, la relation entre le domaine source et le domaine cible (entre le fluide chaud et la colère, le cœur et l'amour, le jeu à risque et la vie), telle que le locuteur l'imagine, est indéterminée, variable sans nul doute d'un locuteur à un autre, et jamais démontrable par les seules manières de parler. Il est nécessaire que je clarifie ce que j'entends par «métaphysique » dans ce contexte. Laissez-moi introduire une distinction entre deux sens du mot, même si la frontière entre les deux est floue inévitablement. Nous faisons des hypothèses implicites sur la nature du monde perçu à travers l'expérience sensible: la solidité des objets "solides", la "lourdeur» de ce qui est en bas et la "hauteur» de ce qui est en haut, les 
représentations de l'espace, du temps, de la cause, etc., du sens commun. Ces présupposés sont, je pense, systématiques et modélisants, ou encore paradigmatiques. Je n'ai aucun doute sur le fait qu'ils varient dans une certaine mesure d'une culture à l'autre (aussi bien que, dans une certaine mesure, d'un individu à l'autre dans telle société particulière); et je ne doute pas qu'ils soient constitués de manière décisive à partir des métaphores conventionnelles et véhiculés par les canaux syntaxiques, sémantiques et idiomatiques du langage. Je qualifierai ces présupposés de métaphysiques en un sens «non chargé » du terme.

À cette signification du qualificatif de métaphysique qui fait référence aux présupposés implicites portant sur ce qui relie en arrière-fond et connecte les éléments du monde perçu par les sens, je souhaite opposer ce que j'appellerai un sens « chargé » du terme. La métaphysique en ce sens constitue une explication (plus ou moins explicitement articulée) de ces aspects du monde qui ne peuvent être perçus par les sens : une théorie explicative de la manière dont le monde travaille qui rende compte des expériences humaines et des conséquences des événements. Une idée métaphysique est du genre de celle que les ethnographes ont forgée de manière caractéristique avec des énoncés du type : «Les $\mathrm{X}$ croient que..${ }^{36} »$.

Nous pouvons illustrer cet écart en référence au soleil qui se « lève » et se « couche » ou à la terre qui est plate. Nous, les modernes "évolués ", quelle que soit l'étendue de nos connaissances en astronomie, vivons notre vie et parlons notre langue comme si le soleil se levait et se couchait réellement, comme si l'univers était plat; c'est là une métaphysique, au sens " non chargé » du terme, qui est sans doute inéluctable pour des créatures qui ont nos cerveaux et nos corps, et qui vivent à la surface de notre planète. Mais il serait absurde pour un ethnographe de l'Angleterre ou des États-Unis d'écrire que "les autochtones croient que la terre est plate et que le soleil s'élève et décline », ou que «les autochtones croient que le cœur est le siège des émotions». Là se situe, par conséquent, le problème de l'anthropologue.

\section{Métaphore conventionnelle et anthropologie de la religion}

Beaucoup d'écrits anthropologiques sur la religion (la plupart des premiers travaux et un bon nombre des analyses contemporaines) intègrent implicitement ou explicitement le présupposé formulé par MacCulloch ${ }^{37}$ : les peuples primitifs partagent des croyances métaphysiques au sens "chargé » du terme, des croyances semblables à celles que les peuples occidentaux ont pu partager autrefois mais plus du tout aujourd'hui. Par suite, lorsque les peuples «primitifs » parlent de leurs émotions dans le langage du cœur, du foie, ou du ventre, ou parlent d'une âme-substance ou du mana, ces manières de parler renferment une métaphysique que nos paroles sur les cœurs brisés et le fait d'avoir toujours de la chance n'impliquent pas du tout. Une telle vue des choses exprime une opposition ethnocentrique entre notre raffinement et leur primitivisme, opposition qui a été dépassée dans la plupart des domaines de l'anthropologie sociale mais qui semble se perpétuer, sous forme déguisée, dans une certaine anthropologie symboliste même récente. "Leurs" manières de parler du monde sont imputées à une pertinence métaphysique ou cosmologique, ce qui n'est pas le cas de nos manières de parler. 

dans lesquelles les arbres ont des esprits à demeure, qui disent qu'elles rencontrent les doubles des morts en rêve, qui pratiquent la divination pour savoir s'il est opportun de monter un raid, ou qui font de la magie ou de la sorcellerie pour le jardinage, ont des visions du monde substantiellement, et même qualitativement, différentes de celles que la plupart des Américains ou des Européens partagent présentement. Mais je doute réellement du présupposé selon lequel de telles notions métaphysiques peuvent être inférées des seules manières de parler, ou que nos métaphores ne représentent pour l'essentiel que des résidus d'anciennes croyances. Nous ne pouvons même pas présupposer que des croyances métaphysiques cohérentes sous-tendent les pratiques magiques ou rituelles, quand bien même celles-ci semblent impliquer de telles croyances ; en témoignent les pieds de lapin et les clubs chanceux. Si nous considérons la métaphore conventionnelle comme un processus universel relevant de la cognition humaine, présent dans toutes les langues (comme les vues de Lakoff et Johnson le laisseraient entendre), alors il se peut qu'il n'y ait jamais eu de lien précis et métaphysiquement pertinent entre un domaine cible et un domaine source. Et bien loin que les métaphores expriment les résidus gelés d'anciennes théories ou théologies populaires, c'est précisément le cycle inverse qui peut souvent se produire. Dans l'histoire d'une langue, un lien entre un domaine source plus accessible en termes d'expérience ou plus facilement caractérisable et un domaine cible moins directement descriptible peut être établi par le biais d'un schème métaphorique; par suite, cette métaphore peut se concrétiser et s'actualiser dans une pratique rituelle; et finalement, une métaphysique peut être créée, élaborant une théorie du domaine ainsi métaphorisé. Une théorie populaire d'un organe corporel compris comme siège physique des émotions peut se former à partir d'une métaphore, mais non l'inverse ; une théorie populaire du succès ou de l'échec conçu comme lié à la présence ou l'absence d'une substance invisible peut se développer à partir d'une métaphore qui s'est concrétisée, qui a été dramatisée dans un rituel et ensuite bricolée en une théologie. C'est précisément ce qui, je le crois, est arrivé, dans certaines régions d'Océanie, avec mana. utilise systématiquement un schème métaphorique conventionnel dans lequel le ventre est le domaine source pour caractériser certaines émotions et certains états cognitifs riches en émotion. "Mon ventre » (ogagu ou bao-gu) est chaud, rouge, mauvais lorsque je suis en colère ; il est bien quand je suis satisfait ; il se rétablit dès que j'ai d'autres pensées ou que je me change les idées; il se rétracte ou se rétrécit lorsque ma colère est apaisée, etc. Je n'ai pas de doute sur le fait que le langage du ventre est pertinent pour les locuteurs Kwaio au même titre que le langage du cœur ou des fluides chauds est pertinent pour nous : il localise les émotions suivant qu'elles sont centrales ou périphériques; il les caractérise dans les termes d'une expérience vivante; il distingue les choses de mon « ventre » de celles qui concernent manatala-gu, «mon intellect ou mon esprit». Mais je ne vois aucune raison, en dehors de ces usages linguistiques, de supposer que le langage du ventre est l'expression d'une théorie populaire du "siège des émotions ", conçu en termes physiques. Combien y a-t-il de populations non occidentales auxquelles les ethnographes ont attribué des croyances portant sur le foie, le ventre, ou le cœur comme "siège des émotions", ou à propos desquels ils ont décrit des notions métaphysiques complexes concernant le mode d'organisation et de contrôle du monde nouménal, croyances et notions dont l'évidence vient de métaphores conventionnelles qui n'ont pas 
une pertinence métaphysique, au sens "chargé » du terme? Un autre exemple Kwaio peut être instructif.

51 Les Kwaio parlent des ancêtres comme étant « chauds » lorsqu'ils accordent des pouvoirs forts ou infligent des sanctions sévères aux êtres vivants, et comme étant "froids " lorsqu'ils ne le font pas. De la même manière, les tombes sont « chaudes » si ce sont des lieux puissants et dangereux de communication avec le monde des esprits, « froides » si elles sont interdites d'accès et abandonnées. Les objets et la magie qui sont mana sont souvent désignés par des verbes d'état qui en termes physiques correspondent à «être chaud» (un modèle très dominant dans plusieurs autres langues Malaita et qui s'étend très loin dans l'ouest de la Mélanésie). En ce qui concerne les Kwaio et leurs voisins, je ne vois aucune évidence pour que le "chaud» de tels objets ou pratiques, êtres ou lieux, dotés de puissance, soit plus qu'une convention métaphorique. L'état des objets physiques soumis à la chaleur (spécialement, en Mélanésie, les pierres à four) est utilisé comme un domaine source, adapté d'une expérience vivante, pour rendre compte de l'efficacité des entités et des actes. Le chaud est invisible, perceptible seulement au toucher, et se manifeste par ses effets (la transformation de la nourriture, du cru au cuit). La « chaleur » des ancêtres, des tombes, des sacra et des formules magiques semblent ne représenter (pour les Kwaio, au moins) aucune notion métaphysique concernant la manière dont les ancêtres produisent réellement des effets dans le monde visible.

Qu'est-ce qui pourrait fournir une preuve de telles croyances? Nous sommes ici sur un terrain glissant. Même si nous en venions à trouver un rituel où, disons, les vivants utilisent le feu ou des objets chauffés dans le but de conduire les ancêtres à faire, quelle que soit la chose, ce qu'ils font, nous ne saurions y voir l'actualisation d'une théologie populaire portant sur ce que les ancêtres font pour produire directement des changements dans le monde de l'expérience humaine, mais simplement une mise en scène de l'homologie métaphorique entre un domaine source et un domaine cible. Avant que le poids de l'évidence ne nous oriente vers un schème métaphysique sous-jacent (en particulier sous la forme de théories populaires cohérentes), mon sentiment est que nous devrions pécher par excès de doute et de prudence ${ }^{38}$. Trop souvent, je le crains, nous avons péché dans l'autre sens.

Notre sauvegarde contre l'imputation fallacieuse de croyances métaphysiques à nos sujets d'enquête, croyances qui semblent impliquées par leurs manières de parler, repose principalement sur le scepticisme et sur la recherche implacable de confirmation de l'évidence en dehors du domaine du langage. Peut-être faut-il aussi mettre en suspens quelques-unes de nos convictions selon lesquelles la pensée, la logique et les cosmologies des peuples non occidentaux sont radicalement différentes les unes des autres et également des nôtres ${ }^{39}$. Malheureusement les possibilités restreintes de terrain prolongé et continu, associées à certaines des habitudes de l'anthropologie symboliste contemporaine, peuvent avoir précisément l'effet inverse. Nous misons très fortement sur notre maitrise des langues de terrain. C'est en partie les intuitions de nos locuteurs indigènes qui pourraient nous dire (si tant est que la question se pose) si leur façon de parler du mana est plus profondément pertinente que notre langage de la chance, si leur langage du «ventre » représente une théorie plus ancrée dans la physiologie que notre langage du cœur. Au moment même où notre quête des structures de sens nous encourage à chercher des connexions cachées et à rendre compte des structures cosmologiques et des équivalences symboliques (et nous récompense ainsi de nos découvertes), la possibilité de jouir de ces années de terrain qui nous permettent de 
commencer à saisir les intuitions des acteurs indigènes, s'évanouit progressivement. Après un total de presque cinq années de terrain chez les Kwaio, étalées sur une période de vingt et un ans, je suis encore loin de comprendre les profondeurs du sens (et les liaisons métaphoriques conventionnelles) du parler Kwaio. [... $]^{40}$.

\section{Orientations à explorer}

Les ethnographes doivent traduire et interpréter au mieux qu'ils le peuvent. En plaidant pour le scepticisme à propos de la lecture en termes de notions théologiques des choses du langage ordinaire et de la pratique rituelle, je m'efforce de donner des arguments pour un engagement plus sérieux dans la réflexion épistémologique sur notre rencontre, et non pour une position de retrait. Certaines des directions de recherche indiquées par le livre de Lakoff et Johnson et par d'autres écrits récents sur la métaphore ${ }^{41}$ sont entièrement différentes. Si toutes les langues incorporent des modèles métaphoriques aussi dominants que ceux dont Lakoff et Johnson esquissent les grandes lignes pour l'anglais et aussi systématiquement interconnectés que ceux que nous montrent Lakoff et Kovecses à propos de nos conceptions de la colère, alors il faut donner au repérage et à l'analyse des schèmes métaphoriques dans les langues non occidentales une grande priorité dans la recherche.

Nous, anthropologues, avons beaucoup à apporter sur ce point, et des écrits récents issus de la tradition symboliste ont marqué quelques progrès dans cette direction. Les contributions à l'ouvrage collectif de Sapir et $\mathrm{Crocker}^{42}$, les études de Fox et de ses collègues sur les métaphores de la parenté et de l'alliance dans l'Indonésie orientale ${ }^{43}$, et les parcours théoriques de Fernandez et Beck ${ }^{44}$, illustrent un engagement croissant de l'anthropologie symboliste dans la question de la métaphore. Certains travaux, tels que l'essai de Salmond ${ }^{45}$ sur les conceptions métaphoriques de la connaissance chez les Anglais et les Maori, se sont nourris directement d'écrits récents relevant de la philosophie et des disciplines apparentées, ce qui est aussi le cas de l'œuvre de Lakoff et Johnson.

La cartographie la plus systématique et la plus ambitieuse des schèmes et relations métaphoriques du langage ordinaire d'une population non occidentale est sans doute celle que nous offre l'analyse de Rosaldo consacrée aux conceptions Ilongot (Philippines) de liget ( « la colère ») et rinata ( « le cœur ») dans leur rapport avec la coutume de la chasse aux têtes. J'ai ailleurs ${ }^{46}$ exprimé un certain scepticisme, conforme à l'argumentaire développé ici, quant à la question de savoir si le parler Ilongot concernant liget et rinata était aussi profondément pertinent et constitutif de l'expérience et de la motivation que le croit Rosaldo. Est-ce que la manière, très différente de la nôtre, dont les Ilongot parlent de la "colère ", implique réellement que la colère et les émotions associées sont sur le plan de l'expérience à ce point différentes de celles que nous caractérisons en termes de fluides chauds? Et est-ce que la façon dont les Ilongot parlent des motifs qui conduisent leurs semblables à capturer des têtes fournit une description analytique adéquate de ces motifs? Notre propre langage psychologique est, de toute évidence, aussi massivement métaphorique; pourrons-nous jamais briser ce cercle, ou devons-nous simplement essayer à la fois de caractériser leurs métaphores et de les traduire dans les nôtres? Le parler des « cœurs » des Ilongot est-il d'une pertinence à ce point supérieure à la nôtre que nous puissions dire, comme le fait Rosaldo ${ }^{47}:$ «Parce que les cœurs peuvent parler comme les personnes et se mouvoir comme la musique ou le vent, les Ilongot 
conceptualisent les "états intérieurs" et les "événements objectifs" dans des termes qui assimilent et relient les événements que nous percevons comme indépendants »? Ou encore, pouvons-nous dire, avec Rosaldo ${ }^{48}$ que : «Ce qui est évident dans le parler des cœurs Ilongot c'est [...] un sens de la dialectique ou de la tension dynamique entre un état de socialité et un état d'opposition et de retrait, entre un moi à l'aise dans son environnement et un moi qui s'isole»?

Je reste un sceptique face à la tendance à conférer aux métaphores conventionnelles des autres peuples une pertinence plus profonde que celle que nous accordons aux nôtres. Jusqu'à ce que nous ayons cartographié les schèmes métaphoriques qui s'expriment dans les langues non occidentales de manière plus systématique que ce qui a été tenté jusqu'à maintenant (en suivant la voie explorée par Rosaldo), et jusqu'à ce que les métaphores de l'anglais et des langues occidentales (et la manière dont elles informent l'expérience et canalisent la pensée) soient plus soigneusement analysées, ces questions doivent rester ouvertes. De telles voies d'exploration devraient représenter pour les anthropologues un défi majeur dans la prochaine décade. Dans cette recherche, il y aura de la place pour ceux qui cherchent à prouver à la fois que les métaphores sont constitutives de l'expérience et guident la pensée en imposant leurs contraintes, et que les schèmes métaphoriques conventionnels des différents peuples varient, la question de notre particularité distinctive et de notre relationnalité métaphorique s'éclairant par là même. Il y aura également place, je le pense, pour le scepticisme. Nous ne pouvons éviter de parler et de penser le monde à travers des réseaux de métaphores conventionnelles. Certaines sont profondes et profondément contraignantes pour notre pensée (même si elles peuvent n'impliquer aucune profondeur métaphysique); pour d'autres (c'est ce que me dit mon intuition) cela est moins évident. Je pense que les "cœurs brisés » ne méritent qu'une observation en passant dans une ethnographie de notre culture. Mais en tant qu'ethnographe travaillant sur des mondes étrangers, et me demandant où placer les parenthèses du doute et le sceau de la certitude, mes propres intuitions culturelles m'offrent peu de confort.

La description des autres cultures qui est le travail essentiel de l'anthropologie et qui remplit les pages des monographies et des revues techniques est beaucoup plus problématique que nous n'en avons conscience. Le respect des données premières, des textes et des contextes, est décisif. Une conscience critique des métaphysiques fallacieuses que véhiculent les interprétations classiques (e.g., " âme-substance », mana, et «l'esprit du don») fournit un fil directeur édifiant. Mais en définitive, le respect de l'évidence apparente à laquelle nous confronte l'ethnographie ne peut que repousser les frontières du doute. La traduction culturelle restera, je le crains, nécessairement problématique.

\section{NOTES}

1. R. M. Keesing, Elota's Story. The Life and Times of a Solomon Islands Big man, St Lucia, Brisbane, University of Queensland Press et New York, St Martin Press, 1978 (réédité à New York, Holt, 
Rinehart and Winson, 1983). R. M. Keesing est l'auteur des autres références présentées dans les notes qui suivent. [NdlR.]

2. R. M. Keesing, Kwaio Religion. The Living and the Dead in a Solomon Island Society, New York, Columbia University Press, 1982. [NdlR.]

3. R. M. Keesing, en collaboration avec P.Corris, Lightning Meets the West Wind. The Malaita Massacre, Melbourne, Oxford University Press, 1980. À propos du meurtre en 1910 d'un administrateur britannique et de la répression qui s'en est suivi, vus par les colonisateurs et les colonisés. [NdlR.]

4. R. M. Keesing, "Ni Geni : Women's Perspectives on Kwaio Society ", in A. M. Strathern, ed., Dealing with Inequality, Cambridge, Cambridge University Press, 1987, et R. M. Keesing, "Kwaio Women Speak: Micro Politics of Autobiography in Solomon Islands Society ", American Anthropologist, 87, 1985, p. 27-39. [NdlR.]

5. R. M. Keesing, Custom and Confrontation. The Kwaio Struggle for Cultural Autonomy, Chicago, University of Chicago Press, 1992. [NdlR.]

6. On trouvera en note la référence aux nombreux articles qui ont précédé et suivi celui que nous présentons ici. Citons entre autres, R.M.Keesing, "Linguistic Knowledge and Cultural Knowledge : Some Doubts and Speculations ", American Anthropologist, 81, 1979, p. 14-36. [NdlR.]

7. Le texte qui suit est la traduction de «Conventional Metaphors and Anthropological Metaphysics : the Problematic of Cultural Translation », Journal of Anthropological Research, 41, 1985, p. 201-217. [NdlR.]

8. Cf. R. M. Keesing, "Anthropology as Interpretive Quest », Current Anthropology, 28, 1987, et le débat qui l'accompagne. [NdlR.]

9. G. Lakoff et M. Johnson, Les métaphores dans la vie quotidienne [1980], Paris, Éd. de Minuit, 1985.

10. D. A. Schon, «Generative Metaphor: A Perspective on Problem-Setting in Social Policy », in A. Ortony, ed., Metaphor and Thought, Cambridge, Cambridge University Press, 1979.

11. D. Holland et N. Quinn, eds, Cultural Models in Language and Thought, New York, Cambridge University Press, 1987.

12. A. Salmond, "Theoretical Landscapes: On Cross-Cultural Conceptions of Knowledge ", in D. Parkin, ed., Semantic Anthropology, Londres, Academic Press, 1982.

13. G. Bateson, La cérémonie du naven [1936], Paris, Éd. de Minuit, 1971.

14. D. Radin, Primitive Man as Philosopher, New York, D. Appleton, 1927. Ogotemmeli était «l'informateur» dogon bien connu mis en scène par M. Griaule dans Dieu d'eau, Muchona the Hornet était celui de Victor W. Turner lors de son terrain chez les Ndembu de Nzambie. [NdT.]

15. R. Brunton, « Misconstrued Order in Melanesian Religion », Man, 15, 1980, p. 112-128.

16. R. Needham, « Skulls and Causality », Man, 11, 1977, p. 71-78.

17. Ibid.

18. R. M. Keesing, « Rethinking Mana », Journal of Anthropological Research, 40, 1984, p. 137-156.

19. Ibid., p. 151-153.

20. L. Oliver Douglas, Ancient Tahitian Society, 3 vols, Cambridge, Harvard University Press, 1974, p. 55.

21. E. S. C. Handy, « Polynesian Religion », Bernice Pauahi Bishop Museum Bulletin, 34, 1927.

22. R. H. Codrington, « Religious Beliefs and Practices in Melanesia », Journal of tbe Anthropological Institute, 10, 1881, p. 261-316; The Melanesians : Studies in Their Religion and Their Folklore, Oxford, Clarendon Press, 1891 ; avec J. Palmer, A Dictionary of tbe Language of Mota, Londres, Society for Promoting Christian Knowledge, 1896.

23. Je suis redevable à R. d'Andrade de m'avoir suggéré ce terme de Campbell (entitivity), mais je ne peux fournir la référence.

24. Cf. F. Steiner, Taboo, Londres, Cohen, 1956.

25. Pour respecter les connotations propres à cette expression typique du langage militaire américain, et éviter les malentendus d'une traduction culturelle confrontée en l'occurrence à 
trois univers de sens, nous avons choisi de conserver pour la suite le terme "off-limits » que l'auteur met lui-même le plus souvent entre guillemets. [NdT.]

26. M. Macintyre fut la première à me suggérer que tapu illustre par excellence les problèmes de réification et de mésinterprétation que j'ai rencontrés avec le terme mana. Dr Macintyre fait remarquer qu'à Tubetube et dans d'autres langues austronésiennes de Milne Bay Province, en Papouasie-Nouvelle-Guinée, souvent les parents disent à un enfant d'abandonner telle activité en s'écriant: "C'est tabu », l'interdit étant uniquement une affirmation temporaire de la volonté parentale. Dr Macintyre poursuit en notant que lorsque les missionnaires chrétiens ont traduit la Bible dans ces langues, ils l'ont de façon significative intitulée Buka Tabu, qui voulait dire, pensaient-ils, "Livre sacré ». En fait, ce titre signifie que certaines personnes ne sont pas autorisées à le lire. La mésinterprétation a conduit à consacrer une nouvelle signification dans les langues indigènes, comme cela s'est souvent vérifié pour mana lorsque le terme a été codifié sous la forme d'une mésinterprétation de passages de la Bible.

27. M. Sahlins, Historical Metaphors and Mythical Realities, Ann Arbor, University of Michigan, 1981.

28. G. Lakoff a montré que les exceptions supposées (comme les locuteurs Quechua qui sont censés inverser ce schème) sont dues habituellement à une mésinterprétation de l'analyste quant à la perspective prise en compte dans les expressions particulières du schème métaphorique.

29. J. Haviland, Body Part Metaphors (Unpub. ms).

30. G. Lakoff et Z. Kovecses, "The Cognitive Model of Anger Inherent in American English ", in D. Holland et N. Quinn, eds, op. cit.

31. M. Rosaldo, Knowledge and Passion. Ilongot Notions of Self and Social Life, Cambridge, Cambridge University Press, 1980.

32. La littérature sur la métaphore, particulièrement en philosophie et dans la critique littéraire, est très étendue; je n'ai aucune prétention à faire la synthèse de cette littérature au profit des anthropologues. Lakoff et Johnson ne sont en aucun cas les premiers à remarquer l'influence de la métaphore dans le langage ordinaire. En réalité, leur livre témoigne d'une vertu d'innocence au regard de cette vaste littérature et du degré d'approfondissement avec lequel les sujets en question ont été explorés par la philosophie continentale, britannique, et américaine. Cette innocence apparente, faut-il le souligner, est trompeuse; M. Johnson a étudié avec Ricœur pendant des années et est très avancé dans la littérature philosophique continentale. Ce que je trouve intéressant sur le plan anthropologique et innovateur sur le plan philosophique dans le travail de Lakoff et Johnson, c'est, d'une part, l'examen qu'ils font de la nature systématique et paradigmatique de la métaphore conventionnelle et, d'autre part, la manière dont ils s'appuient sur l'expérience.

33. M. Rosaldo, op. cit.

34. C. Lutz, " The Domain of Emotion Words on Ifaluk », American Ethnologist, 9, 1982, p. 113-128.

35. J. A. MacCulloch, « Heart », in J. Hastings, ed., Encyclopcedia of Religion and Ethics, Édimbourg, T. \& T. Clark, 1912, p. 557 et 556.

36. Cf. R. Needham, Belief, Language and Experience, Chicago, University of Chicago Press, 1973, sur les problèmes concernant l'attribution d'une « croyance » aux gens.

37. J. A. MacCulloch, op. cit.

38. Je suis très circonspect lorsque de telles articulations sont l'invention d'un philosophe populaire isolé, d'un shaman, d'un savant, ou d'un prêtre de culte.

39. Cf. E. Hutchins, Reasoning on Discourse. An Analysis of Trobriand Land Litigation, Cambridge, Harvard University Press, 1980.

40. Nous nous autorisons à supprimer ici le développement d'un exemple supplémentaire emprunté aux Kwaio qui n'ajoute pas d'élément nouveau à l'argumentaire de l'auteur. [NdT.]

41. Cf. également, A. Ortony, "Metaphor: A Multidimensional Problem », in A. Ortony, ed., op. cit.; P. Ricœur, La métaphore vive, Paris, Seuil, 1975; S. Sacks, ed., On Metaphors, Chicago, University of Chicago Press, 1979. 
42. J. D. Sapir et J. C. Crocker, The Social Use of Metaphor. Essays on the Anthropology of Rhetoric, Philadelphie, University of Pennsylvania Press, 1977.

43. J. J. Fox, ed., The Flow of Life, Cambridge, Harvard University Press, 1980.

44. J. Fernandez, "The Mission of Metaphor in Expressive Culture ", Current Anthropology, 15, 1974, p. 119-146; B. E. F Beck, «The Metaphor as a Mediator between Semantic and Analogic Modes of Thought », Current Anthropology, 19, 1978, p. 83-97.

45. A. Salmond, op. cit.

46. R. M. Keesing, " Anthropology as Interpretive Quest », Current Anthropology, 28, 1987.

47. M. Rosaldo, op. cit., p. 48.

48. Ibid., p. 44.

\section{RÉSUMÉS}

L'examen des travaux récents sur la métaphore conventionnelle associés aux réinterprétations des études classiques portant sur "l'âme-substance » et le mana conduit à confirmer les dangers de la surinterprétation - de l'attribution de théologies et de métaphysiques fictives - chez les ethnographes. Ne sommes-nous pas enclins, dans notre entreprise de traduction culturelle, à prêter aux manières de parler des autres peuples une pertinence qui va bien au-delà de ce qu'elles impliquent en fait?

Recent work on conventional metaphor together with reinterpretations of classic studies of "soul substance" and mana are examined to assess the dangers of overinterpretation - the attribution of inexistent theologies and metaphysics - by ethnographers. In our project of cultural translation, are we prone to attribute deeper salience to other peoples' way of talk than they in fact imply? 